\title{
A MODIFIED MULTIFRACTAL FORMALISM FOR A CLASS OF SELF-SIMILAR MEASURES WITH OVERLAP*
}

\author{
PABLO SHMERKIN ${ }^{\dagger}$
}

Abstract. The multifractal spectrum of a Borel measure $\mu$ in $\mathbb{R}^{n}$ is defined as

$$
f_{\mu}(\alpha)=\operatorname{dim}_{H}\left\{x: \lim _{r \rightarrow 0} \frac{\log \mu(B(x, r))}{\log r}=\alpha\right\} .
$$

For self-similar measures under the open set condition the behaviour of this and related functions is well-understood ([CM92],[Ols95],[AP96]); the situation turns out to be very regular and is governed by the so-called "multifractal formalism". Recently there has been a lot of interest in understanding how much of the theory carries over to the overlapping case; most of the results obtained, however, apply only to a limited range of $\alpha$ (the "left half" of the spectrum). Here we carry out a complete study of the multifractal structure for a class of self-similar measures with overlap which includes the 3-fold convolution of the Cantor measure. Among other things, we prove that the multifractal formalism fails for many of these measures, but it holds when taking a suitable restriction.

Key words. multifractals, self-similarity, overlaps

AMS subject classifications. Primary 28A80; Secondary 28A78

1. Introduction and statement of results. Recall that given a Borel measure $\mu$ in $\mathbb{R}^{n}$, the upper and lower local dimensions of $\mu$ are defined as

$$
\begin{aligned}
& \overline{\operatorname{dim}} \mu(x)=\limsup _{r \rightarrow 0^{+}} \frac{\log \mu(B(x, r))}{\log r} ; \\
& \underline{\operatorname{dim}} \mu(x)=\liminf _{r \rightarrow 0^{+}} \frac{\log \mu(B(x, r))}{\log r} .
\end{aligned}
$$

When $\overline{\operatorname{dim}} \mu(x)=\underline{\operatorname{dim}} \mu(x)$ we refer to the common value as the local dimension of $\mu$ at $x$, and we denote it by $\operatorname{dim} \mu(x)$. One of the main objectives of multifractal analysis is to study the level sets of the (upper or lower) local dimensions of a given measure. To this end a number of "multifractal spectra" are introduced; perhaps the most widely used is

$$
f_{H}(\alpha)=f_{\mu, H}(\alpha)=\operatorname{dim}_{H}\{x \in \operatorname{supp} \mu: \operatorname{dim} \mu(x)=\alpha\},
$$

where $\operatorname{dim}_{H}$ is the Hausdorff dimension. Another basic function in multifractal theory is the $L^{q}$-spectrum, which is defined (for compactly supported measures) as follows: let

$$
S_{\mu, r}(q)=\sup \left\{\sum_{i} \mu\left(B\left(x_{i}, r\right)\right)^{q}:\left\{B\left(x_{i}, r\right)\right\}_{i} \text { is a packing of } \operatorname{supp} \mu\right\} .
$$

The (lower) $L^{q}$-spectrum is then given by

$$
\underline{\tau}(q)=\underline{\tau}_{\mu}(q)=\liminf _{r \rightarrow 0} \frac{\log S_{\mu, r}(q)}{\log r} .
$$

\footnotetext{
*Received January 4, 2004; accepted for publication May 6, 2005.

${ }^{\dagger}$ Department of Mathematics, University of Washington, Seattle, WA 98195-4350, USA (shmerkin@math.washington.edu).
} 
When the limit above exists for all $q$ we speak of "the" $L^{q}$-spectrum $\tau(q)$. The celebrated heuristic principle known as the "multifractal formalism" states that $f_{H}(\alpha)$ and $\tau(q)$ (or $\underline{\tau}(q)$ ) form a Legendre-transform pair. Recall that given a concave function $g(q): \mathbb{R} \rightarrow[-\infty, \infty)$, its Legendre transform is given by

$$
g^{*}(\alpha)=\inf _{q \in \mathbb{R}} q \alpha-g(q) .
$$

It is well-known that $g^{*}$ is also concave, and moreover $g^{* *}=g$. For different accounts and generalizations of the multifractal formalism see [CM92], [Ols95] and [Pes97].

Although false in general, the multifractal formalism has been verified for many natural measures, including self-similar measures under the open set condition ([CM92], [AP96]). Without separation, however, much less is known, and almost all that is known refers to the portion of the $L^{q}$-spectrum corresponding to $q \geq 0$ (or, equivalently, the portion of $f(\alpha)$ corresponding to $\alpha \leq \gamma=\max f$ ); see [LN99] and [PS00] for some of the deep results obtained. In [HL01] a first investigation was made for the case $q<0$; there the authors proved the striking result that for the $m$-fold convolution of the Cantor measure, $m \geq 3$, the maximum of the set of local dimensions is an isolated point.

In this paper we undertake a detailed study of a family of self-similar measures with overlap that includes the 3 -fold convolution of the standard Cantor measure. Our main result is that the multifractal formalism fails for these measures (for an interval of local dimensions, not just the isolated point), but it is verified if we restrict the measure to any subinterval of the support not containing its extreme points, see Theorems 1.5 and 1.6. We also find formulae for the relevant extreme dimensions and prove that the local dimension exists and is almost everywhere constant; some of these expressions are easy to estimate numerically, while others seem to require very heavy computing power. Finally, we investigate some concrete examples which exhibit different multifractal phenomena and suggest future research.

From now on fix integers $d \geq 3$ (the "base") and $m \geq d$ (the "maximum digit"), together with a probability vector $\mathbf{p}=\left(p_{0}, \ldots, p_{m}\right)$. Two related numbers will appear frequently, so we will denote them by special symbols: let

$$
\delta=-1 / \log d,
$$

and

$$
\xi=m /(d-1) .
$$

For technical reasons we will need to impose the following condition on $\mathbf{p}$.

DEFINITION 1. The probability vector $\mathbf{p}$ is said to be regular if

$$
p_{0}, p_{m} \leq p_{i} \text { for all } i=1, \ldots, m-1 .
$$

We will always assume that $\mathbf{p}$ is regular. We remark, however, that most of the results hold under weaker conditions on $\mathbf{p}$, and some results are valid for every probability vector $\mathbf{p}$.

Let $\mu$ be the self-similar measure associated to the weighted iterated function system

$$
\left\{\left(\frac{x}{d}+\frac{i}{d}, p_{i}\right): 0 \leq i \leq m\right\}
$$


In other words, $\mu$ is the only compactly supported Borel probability measure on $\mathbb{R}$ such that $\mu(A)=\sum_{i=0}^{m} p_{i} \mu(d A-i)$ for every Borel set $A$. Another convenient way to look at $\mu$ is as the distribution of the random sums $\sum_{j=0}^{\infty} X_{i} d^{-i}$, where $X_{i}$ takes the value $i$ with probability $p_{i}$. It is not difficult to see that the $k$-fold convolution of the standard Cantor measure can be represented in this way, by taking $d=3, m=k$ and $p_{i}=2^{-k}\left(\begin{array}{c}k \\ i\end{array}\right)$, see Lemma 4.4 .

We will now introduce some notation. For any finite set $\mathcal{A}$ define

$$
\begin{aligned}
& \mathcal{A}^{k}=\left\{\sigma=\left(\sigma_{1}, \ldots, \sigma_{k}\right): \sigma_{j} \in \mathcal{A}\right\} \\
& \mathcal{A}^{*}=\bigcup_{k=0}^{\infty} \mathcal{A}^{k} ; \\
& \mathcal{A}^{\mathbb{N}}=\left\{\sigma=\left(\sigma_{1}, \sigma_{2}, \ldots\right): \sigma_{j} \in \mathcal{A}\right\} \\
& \overline{\mathcal{A}^{*}}=\mathcal{A}^{*} \cup \mathcal{A}^{\mathbb{N}} ; \\
& |\sigma|=\text { length of } \sigma \in \overline{\mathcal{A}^{*}} ; \\
& \sigma \mid k=\text { restriction of } \sigma \in \overline{\mathcal{A}^{*}} \text { to its first } k \text { coordinates; } \\
& T=\text { shift operator on } \mathcal{A}^{\mathbb{N}} ; \\
& \nu_{\mathbf{p}}=\text { Bernoulli (product) measure corresponding to } \mathbf{p}=\left\{p_{i}\right\}_{i \in \mathcal{A}}
\end{aligned}
$$

If $\sigma^{1}, \ldots, \sigma^{j} \in \mathcal{A}^{*}$ we will denote their juxtaposition by $\left(\sigma^{1}, \ldots, \sigma^{j}\right)$. We specialize now to the case $\mathcal{A}=\{0, \ldots, m\}$, and define the "projection" $\pi: \mathcal{A}^{\mathbb{N}} \rightarrow \mathbb{R}$ by

$$
\pi(\omega)=\sum_{i=1}^{\infty} \omega_{i} d^{-i}
$$

A standard fact is that $\mu$ is the projection of $\nu=\nu_{\mathbf{p}}$, in the sense that $\mu(A)=$ $\nu\left(\pi^{-1}(A)\right)$. We will define $\pi$ also for $\sigma \in \mathcal{A}^{*}$; i.e, $\pi(\sigma)=\sum_{i=1}^{|\sigma|} \sigma_{i} d^{-i}$. Observe that $\operatorname{supp} \mu=\pi\left(\mathcal{A}^{\mathbb{N}}\right)=[0, \xi]$, and

$$
\pi\left(\mathcal{A}^{k}\right)=\left\{j d^{-k}: 0 \leq j \leq \sum_{i=1}^{k} m d^{i}\right\} .
$$

If $s \in \mathbb{R}$ let

$$
[s]_{k}=\left\{\sigma \in \mathcal{A}^{k}: \pi(\sigma)=s\right\} .
$$

Note that $[s]_{k}$ is empty unless $s \in \pi\left(\mathcal{A}^{k}\right)$. For $\sigma \in \mathcal{A}^{k}$ let

$$
\mathbf{p}(\sigma)=\prod_{j=1}^{k} p_{\sigma(j)}=\nu\left(\left\{\omega \in \mathcal{A}^{\mathbb{N}}: \omega \mid k=\sigma\right\}\right),
$$

and

$$
\eta(\sigma)=\sum\left\{\mathbf{p}\left(\sigma^{\prime}\right): \sigma^{\prime} \in[\pi(\sigma)]_{k}\right\}=\sum\left\{\mathbf{p}\left(\sigma^{\prime}\right):\left|\sigma^{\prime}\right|=|\sigma|, \pi\left(\sigma^{\prime}\right)=\pi(\sigma)\right\} .
$$

The function $\eta$ will play a key role in what follows. To motivate this, observe that if we define $\mu_{0}=\delta_{0}$ (the Delta measure giving full mass to 0 ), and

$$
\mu_{k+1}(A)=\sum_{i=0}^{m} p_{i} \mu_{k}(d A-i),
$$

then $\mu_{k}$ converges weakly to $\mu$ and it can be easily verified that $\mu_{k}$ assigns $\eta(\sigma)$ mass to $\pi(\sigma)$. Actually, more is true: $\eta(\sigma)$ can be roughly compared to $\mu\left(B\left(\pi(\sigma), d^{-|\sigma|}\right)\right)$; see Proposition 2.3 below. 
Recall that the minimum of the $p_{i}$ is attained at $p_{0}$ or $p_{m}$ (or both). Notice that if we replace $\mathbf{p}$ by $\mathbf{p}^{*}=\left(p_{m}, \ldots, p_{0}\right)$, then the resulting measure $\mu^{*}$ is just a flipped version of $\mu: \mu^{*}(A)=\mu(\xi-A)$ for every $A \subset \mathbb{R}$; thus $\mu$ and $\mu^{*}$ are indistinguishable from the multifractal point of view. Hence we will henceforth assume, without loss of generality, that $p_{0} \leq p_{m} \leq p_{i}$ for $i=1, \ldots, m-1$. Iterating the defining equation $\mu(A)=\sum_{i=0}^{m} p_{i} \mu(d A-i)$ we obtain that $\mu$ is the only probability measure satisfying

$$
\mu(A)=\sum_{\sigma \in \mathcal{A}^{k}} \mathbf{p}(\sigma) \mu\left(d^{k}(A-\pi(\sigma))\right)=\sum_{j=0}^{m+\ldots+m d^{k-1}} \eta\left(\sigma^{j}\right) \mu\left(d^{k} A-j\right),
$$

where $\sigma^{j}$ is a representative of $\left[j d^{-k}\right]_{k}$. Hence if we replace $d$ by $d^{k}, m$ by $m(1+\ldots+$ $d^{k-1}$ ) and $\mathbf{p}$ by $\left\{\eta\left(\sigma^{j}\right)\right\}_{j}$ we get a weighted IFS with the same attractor $\mu$ (it follows easily by induction that the new weights are still regular if $\mathbf{p}$ is regular). By choosing $k=2$ if necessary we can assume that $m>d$; we will do so unless otherwise stated.

We will now introduce a set of transfer matrices. Write

$$
a=1+\left\lfloor\frac{m-d}{d-1}\right\rfloor .
$$

Let us adopt the convention that $p_{i}=0$ for $i \notin\{0, \ldots, m\}$, and define functions $M_{0}, \ldots, M_{m}:\{-a, \ldots, a\}^{2} \rightarrow \mathbb{R}$ by

$$
M_{i}(k, l)=p_{-l d+k+i} .
$$

These functions can be of course considered as matrices in $\mathbb{R}^{(2 a+1) \times(2 a+1)}$. For $\sigma \in \mathcal{A}^{k}$ we will write $M(\sigma)=M_{\sigma(k)} \cdots M_{\sigma(1)}$ (note that $\sigma$ is reversed in the product). The use of appropriately defined transition matrices seems to be a recurrent tool in the investigation of self-similar measures with overlap; see the survey [Lau99] for some instances of this.

We recall some well-known facts and definitions from Linear Algebra. A matrix norm $\|\cdot\|$ is consistent if $\|A B\| \leq\|A\|\|B\|$; all operator norms are consistent. For all consistent norms it is verified that

$$
\rho(A)=\lim _{k \rightarrow \infty}\left\|A^{k}\right\|^{1 / k} \leq\|A\|,
$$

where $\rho(A)$ is the spectral radius of $A$. The generalized spectral radius of a family of matrices $\mathcal{M}$ is defined as

$$
\tilde{\rho}(\mathcal{M})=\limsup _{k \rightarrow \infty}\left(\sup \left\{\rho\left(A_{1} A_{2} \ldots A_{k}\right): A_{i} \in \mathcal{M}\right\}\right)^{1 / k} ;
$$

see [LW95] for a discussion of this and related concepts. Our first result deals with the extreme local dimensions of $\mu$. Let $\bar{\Delta} \mu=\{\overline{\operatorname{dim}} \mu(x): x \in \operatorname{supp} \mu\}$, and define $\underline{\Delta} \mu$ analogously. Let also

$$
\Delta \mu=\{\alpha: \alpha=\operatorname{dim} \mu(x) \text { for some } x \in \operatorname{supp} \mu\} .
$$

Note that $\Delta \mu \subset \bar{\Delta} \mu \cap \underline{\Delta} \mu$, but there is no a priori reason for equality.

THEOREM 1.1. Let

$$
\begin{aligned}
& \underline{\alpha}^{*}=\delta \log \tilde{\rho}\left(M_{0}, \ldots, M_{m}\right) \\
& \alpha^{*}=\delta \log \rho\left(M_{0}, \ldots, M_{m}\right) \\
& \bar{\alpha}=\delta \log p_{0}
\end{aligned}
$$


where

$$
\underline{\rho}\left(M_{0}, \ldots, M_{m}\right)=\inf \left\{\rho(M(\sigma))^{1 /|\sigma|}: \sigma \in \mathcal{A}^{*} \text { and } \sigma_{1} \notin\{0, m\}\right\}
$$

Then:

1. inf $\Delta \mu=\inf \Delta \mu=\underline{\alpha}$, and this common infimum is attained;

2. $\sup \bar{\Delta} \mu=\sup \Delta \mu=\bar{\alpha}$, and this common supremum is attained;

3. $\alpha^{*}$ is the minimum number such that

$$
\Delta \mu \subset\left[\underline{\alpha}, \alpha^{*}\right] \cup\left\{\delta \log p_{m}\right\} \cup\{\bar{\alpha}\}
$$

moreover, the local dimension $\alpha^{*}$ is attained.

4. $\alpha^{*}<\bar{\alpha}$ if $p_{0}<p_{i}$ for all $i=1, \ldots, m-1$.

We remark that the formula for $\bar{\alpha}$ and the interesting fact that $\bar{\alpha}$ is isolated if $p_{0}$ is a strict minimum of the weights are straightforward generalizations of results in [HL01]. On the other hand, the expressions for $\underline{\alpha}$ and $\alpha^{*}$ are new results even for the $m$-fold convolution of the Cantor measure if $m \geq 5$.

We will often need to work with subsets $\Xi_{k}$ of $\mathcal{A}^{k}$ such that the restriction $\left.\pi\right|_{\Xi_{k}}$ is injective, but still $\pi\left(\Xi_{k}\right)=\pi\left(\mathcal{A}^{k}\right)$; this is equivalent to choosing one representative from each nonempty class $\left[j d^{-k}\right]_{k}$. We will henceforth assume that such a family $\left\{\Xi_{k}\right\}_{k \in \mathbb{N}}$ has been selected.

Proposition 1.2. The $L^{q}$ spectrum $\tau(q)$ exists for all $q$. Moreover, if

$$
\bar{S}_{k}(q)=\sum_{\sigma \in \Xi_{k}} \eta(\sigma)^{q},
$$

then $\tau(q)=\lim _{k \rightarrow \infty} \delta k^{-1} \log \bar{S}_{k}(q)$.

We impose now the additional condition $m<2 d-2$ (or, alternatively, $\operatorname{supp} \mu \subset$ $[0,2))$. In this setting sharper and more complete results can be obtained; this is due to the availability of a "barrier digit", which is defined in the next lemma.

Lemma 1.3. Assume that $m<2 d-2$. There exists $\sigma \in \mathcal{A}^{*}$ such that

$$
\pi(\omega)-1<0<\xi<\pi(\omega)+1
$$

for every $\omega \in \mathcal{A}^{\mathbb{N}}$ such that $\omega|| \sigma \mid=\sigma$. If this happens for some $\sigma=(b)$ (a single digit), we will call $b$ a barrier digit .

Proof. Choose $\varepsilon>0$ and $x=\pi(\omega) \in \operatorname{supp} \mu$ such that

$$
\xi-(1+\varepsilon)<x<1-\varepsilon
$$

Take $k$ such that $\sum_{j=k+1}^{\infty} m d^{-j}<\varepsilon / 2$; then $\sigma=\omega \mid k$ verifies the desired property.

Note that if $m<2 d-2$ then for a suitable iteration of the IFS there is a barrier digit, so we will always assume that there is such a digit already in the original IFS. We work with "barrier digits" instead of "barrier words" just for notational convenience. In particular, we stress that although there is no barrier digit for the 3 -fold convolution of the Cantor measure, there is one for some suitable iteration, so our results do apply to this important example. 
The importance of the barrier digit lies in that it allows to restore a weak form of uniqueness in the representation $\pi(\omega)=x$; see Lemma 2.8 in the next section. Suppose that there is a barrier digit $b$, and define

$$
\hat{S}_{k}(q)=\sum_{\sigma \in \Xi_{k-1}} \eta(b, \sigma)^{q} .
$$

$((b, \sigma)$ denotes the concatenation of the digit $b$ and the word $\sigma)$. Observe that $\hat{S}_{k}$ does not depend on the choice of $\Xi_{k}$, since $\pi(\sigma)=\pi\left(\sigma^{\prime}\right)$ implies that $\eta(b, \sigma)=\eta\left(b, \sigma^{\prime}\right)$. We state now our main results.

Theorem 1.4. Assume that $m<2 d-2$. Then

$$
\Delta \mu=\left[\underline{\alpha}, \alpha^{*}\right] \cup\left\{\delta \log p_{m}\right\} \cup\{\bar{\alpha}\} .
$$

TheORem 1.5. Again assume $m<2 d-2$. The following limit exists for all $q$ :

$$
\hat{\tau}(q)=\lim _{k \rightarrow \infty} \delta k^{-1} \log \hat{S}_{k}(q)
$$

Moreover, the limit can be replaced by supremum.

If $K \subset(0, \xi)$ is any closed interval then $\hat{\tau}$ is the $L^{q}$-spectrum of $\left.\mu\right|_{K}$, the restriction of $\mu$ to $K$.

Finally, if $\alpha^{*}=\bar{\alpha}$ then $\tau(q)=\hat{\tau}(q)$ for all $q$, while if $\alpha^{*}<\bar{\alpha}$, then there is $q_{0} \in(-\infty, 0)$ such that

$$
\tau(q)=\left\{\begin{array}{lll}
\bar{\alpha} q & \text { if } & q \leq q_{0} \\
\hat{\tau}(q) & \text { if } & q_{0}<q
\end{array}\right.
$$

This theorem is the key to the understanding of the multifractal formalism for $\mu$ : since the measure has very low concentration near the endpoints of the support, the contribution of one single ball centered at 0 in the sums $S_{r}(q)=\sum_{i} \mu\left(B\left(x_{i}, r\right)\right)^{q}$ is greater than all the others, provided $q$ is sufficiently close to $-\infty$. This instability precludes the multifractal formalism from holding near $\alpha^{*}$, as $\tau(q)$ does not accurately reflect the distribution of the measure near $q=-\infty$. Restricting the measure to a subinterval removes the instability but, thanks to self-similarity, does not alter the "correct" value of the $L^{q}$-spectrum. Hence it is not surprising that $f_{H}(\alpha)$ can be computed as the Legendre transform of $\hat{\tau}(q)$, with the obvious exceptions of the dimensions attained at the extreme points $0, \xi$; this is the content of our next theorem.

THEOREM 1.6. Under the assumption that $m<2 d-2, f_{H}(\alpha)=\hat{\tau}^{*}(\alpha)$ for every $\alpha \in\left(\underline{\alpha}, \alpha^{*}\right)$, where $\hat{\tau}^{*}$ denotes the Legendre transform of $\hat{\tau}$.

An important feature of a multifractal measure which is not implicit in either the multifractal or $L^{q}$ spectra is the existence (or lack thereof) of an almost sure local dimension. This question is answered in the next proposition.

Proposition 1.7. For any $m \geq d$ the local dimension exists and is constant almost everywhere. The almost sure value is given by

$$
\gamma=\inf _{k} \frac{1}{k} \sum_{\sigma \in \Xi_{k}}-\eta(\sigma) \log \eta(\sigma)=\sup _{k} \frac{1}{k} \sum_{\sigma \in \mathcal{A}^{k}}-p(\sigma) \log \|M(\sigma)\| .
$$


where $\|\cdot\|$ is a fixed consistent norm.

We remark that when an almost sure local dimension $\gamma$ exists, many other dimensions of the measure, such as Hausdorff or entropy dimensions, are also equal to $\gamma$.

After a first version of this paper was completed we were informed that KaSing Lau and Xiang-Yang Wang obtained similar results regarding the $L^{q}$ spectrum, albeit using different methods. They give a formula for the $L^{q}$-dimension of the 3 fold convolution of the Cantor measure (the formula is different from ours); it follows from their formula that the $L^{q}$-dimension is analytic except at one point. Their results extend to other overlapping self-similar measures, but in a different direction than the class studied here. We remark that they use the Renewal Theorem and some detailed combinatoric estimates, while our techniques have a more linear algebraic flavor. They also announce results similar to ours regarding the multifractal spectrum, but we have not been able to see their proofs yet.

2. Auxiliary results. This section contains the main technical ingredients of the paper. We begin with a lemma that, although very simple, will play a fundamental role in the sequel.

Lemma 2.1. If $\sigma, \sigma^{\prime} \in \mathcal{A}^{*}$ then

$$
\eta\left(\sigma, \sigma^{\prime}\right) \geq \eta(\sigma) \eta\left(\sigma^{\prime}\right) .
$$

Proof. Follows easily from the definition.

The following proposition is an immediate generalization of [HL01], Lemma 2.1. We include the proof because of its importance and because it relies on the particular structure of the measure $\mu$.

Proposition 2.2. Let $\sigma, \sigma^{\prime} \in \mathcal{A}^{k}$, and suppose that $\sigma$ and $\sigma^{\prime}$ are such that $\left|\pi(\sigma)-\pi\left(\sigma^{\prime}\right)\right|=d^{-k} ;$ i.e. their projections are "neighbors". Then

$$
\frac{1}{k \theta} \leq \frac{\eta(\sigma)}{\eta\left(\sigma^{\prime}\right)} \leq k \theta
$$

where

$$
\theta=\frac{\max _{0 \leq i \leq m} p_{i}}{\min _{0 \leq i \leq m} p_{i}}
$$

Proof. We proceed by induction; the result is clear for $k=1$, so assume it is valid for some $k$, and let $\sigma, \sigma^{\prime} \in \mathcal{A}^{k+1}$. Without loss of generality suppose $\pi\left(\sigma^{\prime}\right)=$ $\pi(\sigma)+d^{-(k+1)}$. Let $A=[\pi(\sigma)]_{k+1}, A^{\prime}=\left[\pi\left(\sigma^{\prime}\right)\right]_{k+1}$. In addition let $B$ be the subset of $A$ of words ending in $m$, and note that $\omega \mapsto\left(\omega_{1}, \ldots, \omega_{k}, \omega_{k+1}+1\right)$ is an injective map from $A \backslash B$ into $A^{\prime}$. Hence

$$
\sum_{\omega \in A \backslash B} \mathbf{p}(\omega) \leq \sum_{\omega \in A \backslash B} \mathbf{p}(\omega \mid k) \theta p_{\omega_{k+1}+1} \leq \theta \sum_{\omega^{\prime} \in A^{\prime}} \mathbf{p}\left(\omega^{\prime}\right)=\theta \eta\left(\sigma^{\prime}\right) .
$$

Now let $\omega \in B$, and write

$$
\pi(\omega)=\pi(\omega \mid k)+m d^{-(k+1)}=j d^{-k}+m d^{-(k+1)} .
$$


Since $\pi(\omega)$ is not maximal in $\pi\left(\mathcal{A}^{k+1}\right), j d^{-k}$ is not maximal in $\pi\left(\mathcal{A}^{k}\right)$. Thus there is $\omega^{\prime} \in \mathcal{A}^{k}$ such that $\pi\left(\omega^{\prime}\right)=(j+1) d^{-k}$. By the inductive hypothesis, $\eta(\omega \mid k) \leq k \theta \eta\left(\omega^{\prime}\right)$, and therefore

$$
\sum_{\omega \in B} \mathbf{p}(\omega)=\eta(\omega \mid k) p_{m} \leq k \theta \eta\left(\omega^{\prime}\right) p_{m} \leq k \theta \eta\left(\omega^{\prime}\right) p_{m-d+1} \leq k \theta \eta\left(\sigma^{\prime}\right),
$$

since

$$
\pi\left(\omega^{\prime}, m-d+1\right)=(j+1) d^{-k}+(m-d+1) d^{-(k+1)}=\pi\left(\sigma^{\prime}\right) .
$$

(it is here that we use regularity; more precisely, that $p_{m} \leq p_{m-d+1}$ ). Now combining (9) and (10) we get

$$
\eta(\sigma)=\sum_{\omega \in A \backslash B} \mathbf{p}(\omega)+\sum_{\omega \in B} \mathbf{p}(\omega) \leq \theta \eta\left(\sigma^{\prime}\right)+k \theta \eta\left(\sigma^{\prime}\right)=(k+1) \eta\left(\sigma^{\prime}\right) .
$$

The other inequality follows in the same way.

Proposition 2.3. For all $\omega \in \mathcal{A}^{\mathbb{N}}$ we have

$$
\overline{\operatorname{dim}} \mu(\pi(\omega))=\limsup _{k \rightarrow \infty} \frac{\delta \log \eta(\omega \mid k)}{k},
$$

and analogously for the lower dimension.

Proof. It follows exactly like in [HL01], Proposition 2.2, using Proposition 2.2 instead of Lemma 2.1 of [HL01].

The following result provides a formula for $\overline{\operatorname{dim}} \mu$ (and $\underline{\operatorname{dim}} \mu$ ) which will play a symmetric role to that of Proposition 2.3.

Proposition 2.4. For every $\omega \in \mathcal{A}^{\mathbb{N}}, \omega \neq(0,0, \ldots)$ or $(m, m, \ldots)$, we have

$$
\overline{\operatorname{dim}} \mu(\pi(\omega))=\limsup _{k \rightarrow \infty} \frac{\delta \log \|M(\omega \mid k)\|}{k}
$$

and analogously for $\underline{\operatorname{dim}} \mu(\pi(\omega))$, where $\|\cdot\|$ denotes any consistent operator.

Proof. Fix $\omega \in \mathcal{A}^{\mathbb{N}}, \omega \neq(0,0, \ldots),(m, m, \ldots)$. Define a sequence $\left\{v_{j}: \mathbb{Z} \rightarrow \mathbb{R}\right\}_{j \in \mathbb{N}}$ by $v_{0}(l)=\delta_{0 l}=1$ if $l=0$ and 0 otherwise; and, for $j \geq 1$,

$$
v_{j}(l)=\sum\left\{\mathbf{p}(\sigma): \sigma \in\left[\pi(\omega \mid j)+l d^{-j}\right]_{j}\right\} .
$$

Write $L=\omega_{j+1}+k$, and observe that for every $n \in \mathbb{Z}$

$$
(\sigma, n) \in\left[\pi(\omega \mid(j+1))+k d^{-(j+1)}\right]_{j+1} \Longleftrightarrow \sigma \in\left[\pi(\omega \mid j)+(L-n) d^{-(j+1)}\right]_{j} ;
$$

and this happens only if $L-n$ is a multiple of $d$ (otherwise the sets involved are empty). Hence, recalling that $p_{n}=0$ if $n \notin\{0, \ldots, m\}$,

$$
\begin{aligned}
v_{j+1}(k) & =\sum\left\{\mathbf{p}(\sigma, n):(\sigma, n) \in\left[\pi(\omega \mid(j+1))+k d^{-(j+1)}\right]_{j+1}\right\} \\
& =\sum_{l \in \mathbb{Z}} p_{(L-l d)} \sum\left\{\mathbf{p}(\sigma): \sigma \in\left[\pi(\omega \mid j)+l d^{-j}\right]_{j}\right\} \\
& =\sum_{l \in \mathbb{Z}} p_{(L-l d)} v_{j}(l) .
\end{aligned}
$$


In the last sum it is enough for $l$ to run from $-a$ to $a$ whenever $-a \leq k \leq a$. Indeed,

$$
\begin{aligned}
p_{(L-l d)} \neq 0 & \Rightarrow 0 \leq \omega_{j+1}+k-l d \leq m \\
& \Rightarrow-m \leq k-l d \leq m \\
& \Rightarrow|l| d \leq m+|k| \leq m+a \\
& \Rightarrow|l| \leq a .
\end{aligned}
$$

Thus we obtain

$$
v_{j+1}(k)=\sum_{l=-a}^{a} M_{\omega_{j+1}}(k, l) v_{j}(l) \quad(-a \leq k, l \leq a) .
$$

Now it follows by induction that $v_{j}=M(\omega \mid j) v_{0}$, where we now consider the $v_{j}$ as vectors of length $2 a+1$ indexed by $(-a, \ldots, a)$ rather than functions from $\mathbb{Z}$ to $\mathbb{R}$. Recalling the definition of $v_{0}$ one sees that the central column of $M(\omega \mid j)$ is precisely $v_{j} ;$ in particular, $M(\omega \mid j)(0,0)=\eta(\omega \mid j)$.

Now observe that if $0<\pi(\omega \mid j)+l d^{-j}<\xi$ then $\left[\pi(\omega \mid j)+l d^{-j}\right]_{j}$ is nonempty, whence $v_{j}(l)>0$. Since $\omega \neq(0,0, \ldots),(m, m, \ldots)$, this shows that there is $j_{0}$ such that $v_{j_{0}}$ contains no zero coordinate. Let

$$
C=\frac{\max _{-a \leq k, l \leq a} M\left(\omega \mid j_{0}\right)(k, l)}{\min _{-a \leq l \leq a} v_{j_{0}}(l)} .
$$

All columns of $M\left(\omega \mid j_{0}\right)$ are bounded by $C v_{j_{0}}$, and therefore it follows from (11) that this happens for every $j \geq j_{0}$. Moreover, Proposition 2.2 implies that

$$
\sum_{l=-a}^{a} v_{j}(l) \leq(2 a+1)(\theta j)^{a} \eta(\omega \mid j)
$$

Now let $\|\cdot\|_{1}$ denote the 1 -norm on $\mathbb{R}^{2 a+1}$. Since all norms are equivalent, it suffices to prove the proposition for the associated operator norm, which is known to be the maximum of the 1-norms of the columns. We have

$$
\eta(\omega \mid j) \leq\left\|v_{j}\right\|_{1} \leq\|M(\omega \mid j)\|_{1} \leq C(2 a+1)(\theta j)^{a} \eta(\omega \mid j) .
$$

Now it is enough to take logarithms, divide by $j$ and recall Proposition 2.3 to complete the proof.

We record an interesting fact that emerged in the previous proof.

Corollary 2.5. Let $K=[a, b]$, with $0<a<b<\xi$. There are constants $C=C(K), D$ such that if $\sigma \in \mathcal{A}^{*}$ and $\pi(\sigma) \in K$, then

$$
\rho(M(\sigma)) \leq\|M(\sigma)\|_{1} \leq C|\sigma|^{D} \eta(\sigma),
$$

where $\|\cdot\|_{1}$ denotes the 1-operator norm.

Proof. The number of consecutive digits 0 or $m$ at the beginning of $\sigma$ is clearly bounded by a constant depending only on $K$. Hence the result follows easily from the proof of the proposition.

We indicate that while $\eta$ is supermultiplicative (in the sense $\eta\left(\sigma, \sigma^{\prime}\right) \geq \eta(\sigma) \eta\left(\sigma^{\prime}\right)$ ), consistent norms are submultiplicative; this fact will be strategically used throughout 
the paper. We underline that the preceding lemma is false for $\omega=(0,0, \ldots)$ if $p_{0}$ is strictly less than the intermediate weights. The reason is that in this case $v_{0}$ has no component in the direction of any eigenvector of $M_{0}$ corresponding to the Perron eigenvalue, so $\left\|M_{0}^{k}\right\|$ cannot be compared with $\left\|M_{0}^{k} v_{0}\right\|$. The same is true of $\omega=(m, m, \ldots)$ if $p_{m}<p_{i}$ for $i=1, \ldots, m-1$. This is another way to look at the distinguished role that the points 0 and $\xi$ play in the multifractal analysis of $\mu$.

Periodic sequences provide the simplest example of points where the local dimension exists and can be computed, as the next lemma shows.

Lemma 2.6. Let $\omega \in \mathcal{A}^{\mathbb{N}}$ be periodic with period $\sigma \in \mathcal{A}^{k}(\sigma \neq(0, \ldots, 0)$ or $(m, \ldots, m))$. Then the local dimension of $\mu$ at $\pi(\omega)$ exists and is given by

$$
\operatorname{dim} \mu(\pi(\omega))=\delta k^{-1} \log \rho(M(\sigma)) .
$$

Proof. Let $\|\cdot\|$ be a consistent norm. Note that $M(\omega \mid k j)=M(\sigma)^{j}$; hence (4) shows that $\|M(\omega \mid k j)\|^{1 / j} \rightarrow \rho(M(\sigma))$, and therefore

$$
\lim _{j \rightarrow \infty} \frac{\delta \log \|M(\omega \mid k j)\|}{k j}=\frac{\delta \log \rho(M(\sigma))}{k} .
$$

Now let $q=k j+r, 0 \leq r<k$. Using the consistency of $\|\cdot\|$ and setting $C=$ $\max \{\|M(\tilde{\sigma})\|:|\tilde{\sigma}|<k\}$ we get

$$
(1 / C)\|M(\omega \mid k(j+1))\| \leq\|M(\omega \mid q)\| \leq C\|M(\omega \mid k j)\| .
$$

This, together with (12), shows that

$$
\lim _{j \rightarrow \infty} \frac{\delta \log \|M(\omega \mid j)\|}{j}=\frac{\delta \rho(M(\sigma))}{k},
$$

which completes the proof. $\square$

Corollary 2.7. $\eta(\sigma) \leq \rho(M(\sigma))$ for every $\sigma \in \mathcal{A}^{*}$.

Proof. Let $\omega \in \mathcal{A}^{\mathbb{N}}$ be periodic with period $\sigma$. Since, by Lemma 2.1, $j \log \eta(\sigma) \leq$ $\log \eta(\omega \mid j \sigma)$, we obtain

$$
\frac{\delta \log \rho(M(\sigma))}{|\sigma|}=\lim _{j \rightarrow \infty} \frac{\delta \log \eta(\omega|j| \sigma \mid)}{j|\sigma|} \geq \frac{\delta \log \eta(\sigma)}{|\sigma|} .
$$

From here the corollary follows immediately.

The following lemma illustrates the main advantage of the existence of barrier digits; compare with Lemma 2.1 .

Lemma 2.8. For all $\sigma, \sigma^{\prime} \in \mathcal{A}^{*}$ for which $\sigma_{1}^{\prime}$ is a barrier digit, we have

$$
\eta\left(\sigma, \sigma^{\prime}\right)=\eta(\sigma) \eta\left(\sigma^{\prime}\right)
$$

Proof. Take $\omega, \omega^{\prime} \in \mathcal{A}^{*}$ such that $|\omega|=|\sigma|,\left|\omega^{\prime}\right|=\left|\sigma^{\prime}\right|$ and $\pi\left(\omega, \omega^{\prime}\right)=\pi\left(\sigma, \sigma^{\prime}\right)$. Therefore

$$
\left|\pi\left(\omega^{\prime}\right)-\pi\left(\sigma^{\prime}\right)\right|=d^{|\omega|}|\pi(\omega)-\pi(\sigma)| .
$$


Hence either $\left|\pi\left(\omega^{\prime}\right)-\pi\left(\sigma^{\prime}\right)\right| \geq 1$ or $\pi\left(\omega^{\prime}\right)=\pi\left(\sigma^{\prime}\right)$. The first is impossible since $\sigma_{1}^{\prime}$ is a barrier digit, so we must have $\pi\left(\omega^{\prime}\right)=\pi\left(\sigma^{\prime}\right)$ and $\pi(\omega)=\pi(\sigma)$. But then

$$
\begin{aligned}
\eta\left(\sigma, \sigma^{\prime}\right) & =\sum\left\{\mathbf{p}(\omega) \mathbf{p}\left(\omega^{\prime}\right): \pi\left(\omega, \omega^{\prime}\right)=\pi\left(\sigma, \sigma^{\prime}\right),|\omega|=|\sigma|,\left|\omega^{\prime}\right|=\left|\sigma^{\prime}\right|\right\} \\
& =\sum\{\mathbf{p}(\omega): \pi(\omega)=\pi(\sigma)\} \sum\left\{\mathbf{p}\left(\omega^{\prime}\right): \pi\left(\omega^{\prime}\right)=\pi\left(\sigma^{\prime}\right)\right\} \\
& =\eta(\sigma) \eta\left(\sigma^{\prime}\right) . \quad
\end{aligned}
$$

Recall (6) and note that $\hat{S}_{k}$ is a strictly decreasing function such that $\hat{S}_{k}(0)=$ $\# \Xi_{k}>1$ and $\hat{S}_{k}(1)<\sum_{\sigma \in \Xi_{k}} \eta(\sigma)=1$. Hence there is a unique "auxiliary exponent" $0<\beta_{k}<1$ such that $\hat{S}_{k}\left(\beta_{k}\right)=1$.

Lemma 2.9. $\lim _{k \rightarrow \infty} \beta_{k}=1$.

Proof. Using Lemma 2.1 once more we get $\eta(b, \sigma) \geq p_{b} \eta(\sigma)$. Hence

$$
1 \geq p_{b}^{\beta_{k}} \sum_{\sigma \in \Xi_{k-1}} \eta(\sigma)^{\beta_{k}}>p_{b} \max _{\sigma \in \Xi_{k-1}} \eta(\sigma)^{\beta_{k}-1},
$$

where for the second inequality we used that $\sum_{\sigma \in \Xi_{k-1}} \eta(\sigma)=1$. Note that

$$
\lim _{k \rightarrow \infty} \max _{\sigma \in \Xi_{k-1}} \eta(\sigma)=0 .
$$

There are several ways to see this. For instance, we know from the proof of Proposition 2.4 that

$$
\eta(\sigma) \leq\|M(\sigma)\|_{1} \leq\left(\max \left\{\|M(i)\|_{1}\right\}\right)^{|\sigma|},
$$

where $\|M(i)\|_{1}$ is the maximum of the 1-norms of the columns of $M_{i}$, whose non-zero coordinates are some, but not all, of the $p_{i}$. Hence $\eta(\sigma)<c^{|\sigma|}$ for some $c<1$, and this establishes (14) which, together with (13), imply the lemma.

\section{Proof of the main results.}

Proof of Theorem 1.1. Let $\left\{\omega^{j}\right\}$ be a sequence in $\mathcal{A}^{\mathbb{N}}$ such that $\underline{\operatorname{dim}} \mu\left(\pi\left(\omega^{j}\right)\right) \rightarrow$ $\inf \Delta \mu$ as $j \rightarrow \infty$. For each $j$ choose $k_{j}$ such that

$$
\frac{\delta \log \eta\left(\omega^{j} \mid k_{j}\right)}{k_{j}}<\underline{\operatorname{dim}} \mu\left(\pi\left(\omega^{j}\right)\right)+1 / j
$$

Let $\omega^{\prime j}$ be the periodic sequence with period $\omega^{j} \mid k_{j}$. It follows from Lemma 2.6 and Corollary 2.7 that $\operatorname{dim}_{\mu}\left(\pi\left(\omega_{j}^{\prime}\right)\right)$ exists and

$$
\operatorname{dim}_{\mu}\left(\pi\left(\omega_{j}^{\prime}\right)\right)=\frac{\delta \log \rho\left(M\left(\omega^{j} \mid k_{j}\right)\right)}{k_{j}} \leq \frac{\delta \log \eta\left(\omega^{j} \mid k_{j}\right)}{k_{j}} .
$$

Together with (15)this proves that inf $\Delta \mu=\inf \underline{\Delta} \mu$.

We will now show that this common infimum is attained. As before, let $\omega^{\prime j}$ be a sequence of periodic sequences such that $\operatorname{dim} \mu\left(\pi\left(\omega^{\prime j}\right)\right) \rightarrow \inf \Delta \mu$. By compactness we may assume that $\omega^{\prime j}$ is convergent and, moreover, monotone in the sense that the period of $\omega^{\prime j+1}$ starts with the period of $\omega^{\prime j}$ for all $j$. Let $\omega^{\prime}$ denote the limiting 
sequence, and write $k_{j}$ for the length of the period of $\omega^{\prime j}$. By virtue of Corollaries 2.5 and 2.7 we have

$$
\liminf _{j \rightarrow \infty} \delta k_{j}^{-1} \eta\left(\omega^{\prime} \mid k_{j}\right)=\lim _{j \rightarrow \infty} \delta k_{j}^{-1} \rho\left(\omega^{\prime} \mid k_{j}\right)=\inf \Delta \mu,
$$

whence $\underline{\operatorname{dim}} \mu\left(\pi\left(\omega^{\prime}\right)\right)=\inf \underline{\Delta} \mu=\inf \Delta \mu$.

Now let $k \in \mathbb{N}$ and $M=M(\sigma)$ for some $\sigma \in \mathcal{A}^{k}$. Let $\omega \in \mathcal{A}^{\mathbb{N}}$ be periodic with period $\sigma$. It follows from Lemma 2.6 that inf $\Delta \mu \leq \delta k^{-1} \rho(M)$. Hence

$$
\inf \Delta \mu \leq \delta \log \tilde{\rho}\left(M_{0}, \ldots, M_{m}\right) .
$$

For the other inequality fix $\varepsilon>0$. The previously stated facts about periodic sequences and Lemma 2.6 show the existence of $\sigma \in \mathcal{A}^{*}$ such that

$$
\delta|\sigma|^{-1} \log \rho(M(\sigma))<\inf \Delta \mu+\varepsilon .
$$

Since $|\sigma|$ can be arbitrarily large we conclude that

$$
\delta \log \tilde{\rho}\left(M_{0}, \ldots, M_{m}\right) \leq \inf \Delta \mu .
$$

From Proposition 2.3 it immediately follows that

$$
\operatorname{dim} \mu(0)=\delta \log p_{0} ; \quad \operatorname{dim} \mu(\xi)=\delta \log p_{m} .
$$

Since $\eta(\sigma) \geq \mathbf{p}(\sigma)$ and $\mathbf{p}$ is regular, $\overline{\operatorname{dim}} \mu(x) \leq \bar{\alpha}$ for all $x \in[0, \xi]$.

To show that $\bar{\alpha}$ is isolated in $\bar{\Delta} \mu$ if $p_{0}<p_{i}$ for $i=1, \ldots, m-1$, we can proceed exactly like in [HL01], Theorem 1.1; we therefore omit the details.

It remains to prove (5). It follows from Proposition 2.4 and submultiplicativity of consistent norms that $\overline{\operatorname{dim}} \mu(\pi(T \sigma)) \geq \overline{\operatorname{dim}} \mu(\pi(\sigma))$. Note that every $x \in(0, \xi)$ can be represented by a sequence containing at least one digit other than 0 and $m$ (it has to be different from $(0,0, \ldots)$ and $(m, m, \ldots)$; replace any occurrence of $(0, m)$ by $(1, m-d)$ and any occurrence of $(m, 0)$ by $(m-1, d))$. Therefore by taking such a sequence and shifting it we see that

$$
\sup (\Delta \mu \backslash\{\operatorname{dim} \mu(0), \operatorname{dim} \mu(\xi)\})=\sup \left\{\overline{\operatorname{dim}} \mu(\pi(\omega)): \omega \in \mathcal{A}^{\mathbb{N}}, \omega_{1} \notin\{0, m\}\right\} .
$$

Now we can proceed as above, but now using Proposition 2.4 instead of Proposition 2.3, to show that in the supremum above we can restrict ourselves to periodic sequences. The result then follows from Lemma 2.6.

The formulae for $\underline{\alpha}$ and $\alpha^{*}$ given in Theorem 1.1 are not very useful unless there is some way to estimate their value in concrete cases. This is a difficult problem, but we nevertheless have the following corollary.

Corollary 3.1. Fix a consistent matrix norm $\|\cdot\|$, and let

$$
\begin{aligned}
& \tilde{\rho}_{k}=\max \left\{\rho(M(\sigma)): \sigma \in \mathcal{A}^{k}\right\}^{1 / k} ; \\
& \hat{\rho}_{k}=\max \left\{\|M(\sigma)\|: \sigma \in \mathcal{A}^{k}\right\}^{1 / k} ; \\
& \tilde{\rho}_{k}^{*}=\min \left\{\rho(M(\sigma)): \sigma \in \mathcal{A}^{k}, \sigma_{1} \notin\{0, m\}\right\}^{1 / k} ; \\
& \hat{\rho}_{k}^{*}=\min \left\{\eta(\sigma): \sigma \in \mathcal{A}^{k}, \sigma_{1} \notin\{0, m\}\right\}^{1 / k} .
\end{aligned}
$$

Then

$$
\begin{aligned}
& \delta \log \hat{\rho}_{k} \leq \underline{\alpha} \leq \delta \log \tilde{\rho}_{k} \\
& \delta \log \tilde{\rho}_{k}^{*} \leq \alpha^{*} \leq \delta \log \hat{\rho}_{k}^{*},
\end{aligned}
$$


for every $k$. Moreover,

$$
\begin{aligned}
& \lim _{k \rightarrow \infty} \delta \log \hat{\rho}_{k}=\lim _{k \rightarrow \infty} \delta \log \tilde{\rho}_{k}=\underline{\alpha} ; \\
& \lim _{k \rightarrow \infty} \delta \log \hat{\rho}_{k}^{*}=\lim _{k \rightarrow \infty} \delta \log \tilde{\rho}_{k}^{*}=\alpha^{*} .
\end{aligned}
$$

Proof. Let $\mathcal{M}=\left\{M_{0}, \ldots, M_{m}\right\}$. The inequalities $\tilde{\rho}_{k} \leq \tilde{\rho}(\mathcal{M}) \leq \hat{\rho}_{k}$ follow easily from (4). The fact that $\hat{\rho}_{k}-\tilde{\rho}_{k} \rightarrow 0$ as $k \rightarrow \infty$ was proved in [BW92] for any bounded set of matrices; hence

$$
\lim _{k \rightarrow \infty} \delta \log \hat{\rho}_{k}=\lim _{k \rightarrow \infty} \delta \log \tilde{\rho}_{k}=\underline{\alpha} .
$$

Now we turn to the approximations of $\alpha^{*}$. From (4), Corollary 2.5 and Corollary 2.7 we obtain

$$
\eta(\sigma) \leq \rho(\sigma) \leq C|\sigma|^{D} \eta(\sigma),
$$

for every $\sigma \in \mathcal{A}^{*}$ such that $\sigma_{1} \notin\{0, m\}$, where $C$ and $D$ are independent of $\sigma$. From here it follows that

$$
\delta \log \tilde{\rho}_{k}^{*} \leq \alpha^{*} \leq \delta \log \hat{\rho}_{k}^{*} \leq \delta \log \tilde{\rho}_{k}^{*}-\delta \frac{\log \left(C k^{D}\right)}{k} .
$$

In particular,

$$
\lim _{k \rightarrow \infty} \delta \log \hat{\rho}_{k}^{*}=\lim _{k \rightarrow \infty} \delta \log \tilde{\rho}_{k}^{*}=\alpha^{*}
$$

Proof of Proposition 1.2. Let $\mu_{k}$ be the discrete measure assigning mass $\eta(\sigma)$ to $\pi(\sigma)$ for $\sigma \in \mathcal{A}^{k}$. Note that, for $x \in \operatorname{supp} \mu$,

$$
\mu\left(B\left(x, d^{-k}\right)\right) \leq \mu_{k}\left(B\left(x, c d^{-k}\right)\right) \leq \mu\left(B\left(x, 2 c d^{-k}\right)\right),
$$

where $c=1+\operatorname{diam}(\operatorname{supp} \mu)=1+\xi$. Let $x=\pi(\omega)$, and observe that $\pi(\omega \mid k) \in$ $B\left(x, c d^{-k}\right)$. On the other hand, $B\left(x, c d^{-k}\right)$ contains no more than $\lfloor 2 c\rfloor$ consecutive points of $\pi\left(\mathcal{A}^{k}\right)$. Hence, by Proposition 2.2,

$$
\eta(\omega \mid k) \leq \mu_{k}\left(B\left(x, c d^{-k}\right)\right) \leq\lfloor 2 c\rfloor(\theta k)^{\lfloor 2 c\rfloor} \eta(\omega \mid k) .
$$

From (16) and (17) a routine, but maybe a little bit tedious, calculation shows that

$$
\underline{\underline{\tau}}(q)=\liminf _{k \rightarrow \infty} \frac{\delta \log \bar{S}_{k}(q)}{k} .
$$

We will now show that the limit in (18) exists. Write

$$
\Xi_{n, k}^{*}=\left\{(\sigma, \omega): \sigma \in \Xi_{n}, \omega \in \Xi_{k}\right\} \subset \mathcal{A}^{k+n} .
$$

Note that $\pi\left(\Xi_{n, k}^{*}\right)=\pi\left(\mathcal{A}^{k+n}\right)$. On the other hand, if $\pi(\sigma, \omega)=\pi\left(\sigma^{\prime}, \omega^{\prime}\right)$ for $\sigma, \sigma^{\prime} \in$ $\Xi_{n} ; \omega, \omega^{\prime} \in \Xi_{k}$, then

$$
\left|\pi(\sigma)-\pi\left(\sigma^{\prime}\right)\right|=d^{-n}\left|\pi(\omega)-\pi\left(\omega^{\prime}\right)\right| \leq d^{-n} \xi .
$$


Hence at most $\lfloor\xi\rfloor+1$ elements of $\Xi_{n, k}^{*}$ project onto the same number. Using once again Lemma 2.1 we obtain

$$
\bar{S}_{n}(q) \bar{S}_{k}(q) \leq \sum_{\sigma \in \Xi_{n}, \tau \in \Xi_{k}} \eta(\sigma, \tau)^{q} \leq(\lfloor\xi\rfloor+1) \bar{S}_{n+k}(q)
$$

for $q \geq 0$, and

$$
\bar{S}_{n}(q) \bar{S}_{k}(q) \geq \sum_{\sigma \in \Xi_{n}, \tau \in \Xi_{k}} \eta(\sigma, \tau)^{q} \geq \bar{S}_{n+k}(q)
$$

for $q \leq 0$. In either case, sub/supermultiplicativity shows that the limit in (18) exists. $\square$

Two remarks are in order. First, Peres and Solomyak [PS00] have proved that for any self-similar measure the $L^{q}$-spectrum exists in the range $q \geq 0$, regardless of separation; their proof also relies on submultiplicativity. Second, Lau and Ngai showed in [LN99] that the Legendre transform of $\underline{\tau}_{\mu}(q)$ is always an upper bound for $f_{\mu, H}(\alpha)$.

We begin now our investigation of the case $m<2 d-2$. Recall that by iterating the IFS if necessary we can assume without loss of generality the existence of a barrier digit $b$.

Proof of theorem 1.4. It is clear that $\operatorname{dim} \mu(0)=\delta \log p_{0}$ and $\operatorname{dim} \mu(\xi)=\delta \log p_{m}$. Hence it suffices to prove that $\left(\underline{\alpha}, \alpha^{*}\right) \subset \Delta \mu$.

Fix $k \in \mathbb{N}$, and let $\mu^{k}$ be the attractor of the IFS

$$
\left\{\left(d^{-k}\left(x+d^{k} \pi(b, \sigma)\right), \eta(b, \sigma)^{\beta_{k}}\right): \sigma \in \Xi_{k-1}\right\},
$$

where $\beta_{k}$ is the auxiliary exponent defined before Lemma 2.9 .

Note that, since $b$ is a barrier, $0<\pi(b, \sigma)<1$. Thus the maps are of the form $d^{-k}(x+j)$, with $0<j<d^{k}$, and the IFS verifies the strong separation condition. This allows us to use the multifractal theory developed in [CM92]; see also [Fal97], Chapter 11. Let

$$
\mathcal{A}_{k}=\left\{(b, \sigma): \sigma \in \Xi_{k-1}\right\} .
$$

Endow $\mathcal{A}_{k}^{\mathbb{N}}$ with the Bernoulli measure $\nu^{k}$ for the weights $\eta(b, \sigma)^{\beta_{k}}$, and denote by $\pi^{k}: \mathcal{A}_{k}^{\mathbb{N}} \rightarrow \mathbb{R}$ the canonical projection. Observe that

$$
\overline{\operatorname{dim}} \mu^{k}\left(\pi^{k}(\omega)\right)=\limsup _{j \rightarrow \infty} \frac{\delta \log \nu^{k}([\omega \mid j])}{j k}
$$

and analogously for the lower dimension, where $[\omega \mid j]$ denotes the cylinder generated by $\omega \mid j$. There is a canonical map $\phi^{k}: \mathcal{A}_{k}^{\mathbb{N}} \rightarrow \mathcal{A}^{\mathbb{N}}$; clearly $\pi \circ \phi^{k}=\pi^{k}$. Moreover,

$$
\nu^{k}([\omega \mid j])=\eta\left(\omega_{1}\right)^{\beta_{k}} \cdots \eta(\omega \mid j)^{\beta_{k}}=\eta\left(\phi^{k}(\omega) \mid k j\right)^{\beta_{k}},
$$

where for the last equality we used Lemma 2.8. From this, (20) and Proposition 2.3 it follows that if $x=\pi^{k}(\omega)$ for some $\omega \in \mathcal{A}_{k}^{\mathbb{N}}$, then

$$
\overline{\operatorname{dim}} \mu^{k}(x)=\beta_{k} \overline{\operatorname{dim}} \mu(x),
$$


and analogously for the lower dimension. This equality is the key to estimating the multifractal spectrum of $\mu$, by reducing it to the study of the spectrum of the measures $\mu^{k}$ (which are well-understood). As a first instance of this, note that $\Delta \mu \supset \beta_{k}^{-1} \Delta \mu^{k}$. Hence we obtain (see [CM92]) $\Delta \mu \supset\left[\underline{\alpha}_{k}, \bar{\alpha}_{k}\right]$, where

$$
\begin{aligned}
\underline{\alpha}_{k} & =\frac{\delta \max _{\sigma \in \Xi_{k-1}} \log \eta(b, \sigma)}{\beta_{k} k} ; \\
\bar{\alpha}_{k} & =\frac{\delta \min _{\sigma \in \Xi_{k-1}} \log \eta(b, \sigma)}{\beta_{k} k} .
\end{aligned}
$$

Letting $k$ run through the positive integers we get $\Delta \mu \supset\left(\inf _{k} \underline{\alpha}_{k}, \sup _{k} \bar{\alpha}_{k}\right)$. To complete the proof we will now show that $\inf _{k} \underline{\alpha}_{k}=\underline{\alpha}$ and $\sup _{k} \bar{\alpha}_{k}=\alpha^{*}$. We have that $\underline{\alpha} \leq \underline{\alpha}_{k}$ for all $k$; hence it suffices to show that $\liminf _{k \rightarrow \infty} \underline{\alpha}_{k} \leq \underline{\alpha}$. Since $\eta(b, \sigma) \geq p_{b} \eta(\sigma)$ we have

$$
\underline{\alpha}_{k} \leq \frac{\delta \log p_{b}+\delta \max _{\sigma \in \Xi_{k-1}} \log \eta(\sigma)}{\beta_{k} k} .
$$

Thus, using Lemma 2.9,

$$
\liminf _{k \rightarrow \infty} \underline{\alpha}_{k}=\liminf _{k \rightarrow \infty} \delta k^{-1} \max _{\sigma \in \Xi_{k}} \log \eta(\sigma) \leq \underline{\alpha} .
$$

For the other equality, $\sup _{k} \bar{\alpha}_{k}=\alpha^{*}$, observe that, since $\overline{\operatorname{dim}} \mu(\pi(b, \sigma)) \geq \overline{\operatorname{dim}} \mu(\pi(\sigma))$ (by Proposition 2.4),

$$
\alpha^{*}=\sup _{\omega} \overline{\operatorname{dim}} \mu(\pi(b, \omega)) \leq \delta \sup _{k} k^{-1} \min _{\sigma \in \Xi_{k-1}} \log \eta(b, \sigma) \leq \sup _{k} \bar{\alpha}_{k} .
$$

This concludes the proof.

Proof of Theorem 1.5. To make the proof easier to read we will split it into three parts.

First step. We begin by showing that the limit in the definition of $\hat{\tau}$ exists. Note that if we write

$$
\Xi_{k, n}^{*}=\left\{\left(\sigma, b, \sigma^{\prime}\right): \sigma \in \Xi_{k-1}, \sigma^{\prime} \in \Xi_{n-1}\right\},
$$

then $\pi$ restricted to $\Xi_{k, n}^{*}$ is injective (this follows from $b$ being a barrier digit), and

$$
\left\{\eta(b, \omega): \omega \in \Xi_{k, n}^{*}\right\} \subset\left\{\eta(b, \sigma): \sigma \in \Xi_{k+n-1}\right\} .
$$

Hence we have

$$
\begin{aligned}
\hat{S}_{k}(q) \hat{S}_{n}(q) & =\sum\left\{\eta\left(b, \sigma, b, \sigma^{\prime}\right)^{q}: \sigma \in \Xi_{k}, \sigma^{\prime} \in \Xi_{n}\right\} \\
& =\sum\left\{\eta(b, \omega)^{q}: \omega \in \Xi_{k, n}^{*}\right\} \\
& \leq \hat{S}_{k+n}(q),
\end{aligned}
$$

where for the first equality we again used Lemma 2.8, and for the last inequality we used (22). 
Therefore the sequence $\log \hat{S}_{n}(q)$ is superadditive, whence the limit of $a_{k}=$ $\delta k^{-1} \log \hat{S}_{k}(q)$ exists and is equal to $\sup _{k} a_{k}$.

Second step. The estimates used in establishing Proposition 1.2 also show the following: if $K$ is any closed subinterval of $[0, \xi]$ and

$$
S_{j}^{K}(q)=\sum\left\{\eta(\sigma)^{q}: \sigma \in \Xi_{k} \text { and } \pi(\sigma) \in K\right\},
$$

then

$$
\underline{\tau}^{K}(q)=\liminf _{k \rightarrow \infty} \delta k^{-1} \log S_{j}^{K}(q),
$$

where $\underline{\tau}^{K}$ denotes the lower $L^{q}$-spectrum of $\left.\mu\right|_{K}$; and an analogous assertion holds for the upper limit.

Choose any $\sigma \in \mathcal{A}^{*}$ such that for any $\omega \in \mathcal{A}^{\mathbb{N}}$ beginning with $\sigma, \pi(\omega) \in K$. Let $k=|\sigma|$. For all $j>k+1$ we have

$$
\begin{aligned}
S_{j}^{K}(q) & \geq \sum\left\{\eta\left(\sigma, b, \sigma^{\prime}\right)^{q}: \sigma^{\prime} \in \Xi_{j-k-1}\right\} \\
& =\eta(\sigma)^{q} \hat{S}_{j-k}(q)
\end{aligned}
$$

by Lemma 2.8. From here we obtain

$$
\limsup _{k \rightarrow \infty} \delta k^{-1} \log S_{j}^{K}(q) \leq \hat{\tau}(q) .
$$

For the opposite inequality we consider the cases $q \geq 0$ and $q<0$ separately. For nonnegative $q$ we have $\eta(\sigma)^{q} \leq p_{b}^{-q} \eta(b, \sigma)^{q}$, whence

$$
S_{j}^{K}(q) \leq p_{b}^{-q} \hat{S}_{j}(q) .
$$

For negative $q$ we use Corollary 2.5; at this point we need to assume that $K$ is bounded away from 0 and $\xi$. From the Corollary we get

$$
\begin{aligned}
\eta(b, \sigma) & \leq\|M(b, \sigma)\| \\
& \leq\left\|M_{b}\right\|\|M(\sigma)\| \\
& \leq\left\|M_{b}\right\| C|\sigma|^{D} \eta(\sigma)
\end{aligned}
$$

where $C$ and $D$ do not depend on $\sigma$ (nor $|\sigma|$ ). Therefore for $q<0$ we obtain

$$
\eta(\sigma)^{q} \leq C^{\prime}|\sigma|^{-q D} \eta(b, \sigma)^{q},
$$

where $C^{\prime}$ does not depend on $\sigma$. Hence

$$
S_{j}^{K}(q) \leq C^{\prime} j^{-q D} \hat{S}_{j}(q) .
$$

From (24) and (25) we conclude

$$
\liminf _{j \rightarrow \infty} \delta j^{-1} \log S_{j}^{K}(q) \geq \hat{\tau}(q) .
$$

This together with (23) shows that the $L^{q}$ spectrum of $\left.\mu\right|_{K}$ exists and is equal to $\hat{\tau}$, as desired. 
Before proceeding to the next step, let us remark that the fact that $\hat{\tau}$ is the $L^{q}$ spectrum of $\left.\mu\right|_{K}$ guarantees that $\hat{\tau}$ is a concave (hence continuous) increasing function, and that $\hat{\tau}(0)=-1$; see [LN99], Proposition 2.3 (all these facts can also be checked directly from the definition).

Third step. We will now prove (8). The case $q \geq 0$ follows like in the previous step, so we will assume $q<0$. Let

$$
\begin{array}{ll}
K_{1}=\left[0, d^{-1}\right] ; & K_{1}^{\prime}=\left[\xi-d^{-1}, \xi\right] ; \\
K_{2}=\left[d^{-1}, 1\right] ; & K_{2}^{\prime}=\left[\xi-1, \xi-d^{-1}\right] ; \\
K_{3}=\left[d^{-1}, \xi-d^{-1}\right] ; & K_{4}=K_{2} \cap K_{2}^{\prime} .
\end{array}
$$

We will keep using the notation $S_{j}^{K}(q)$ (even if $K$ contains 0 or $\xi$ ). Let us adopt the convention that $S_{0}^{K}(q)=1$. Note that

$$
\bar{S}_{j}(q)=S_{j}^{K_{1}}(q)+S_{j}^{K_{3}}(q)+S_{j}^{K_{1}^{\prime}}(q)
$$

(there is a minor issue with the points 1 and $\xi-1$, but this does not affect the argument). Moreover, if $d^{-(k+1)} \leq \pi(\sigma)<d^{-k}$ for $\sigma \in \mathcal{A}^{*}$, then $\sigma$ begins with at least $k$ zeros, whence $\eta(\sigma)=p_{0}^{k} \eta\left(\sigma^{\prime}\right)$ for $\sigma^{\prime}=T^{k} \sigma$. Moreover, $\left|\sigma^{\prime}\right|=\sigma-k$ and $d^{-1} \leq \pi\left(\sigma^{\prime}\right)<1$. Hence

$$
S_{j}^{K_{1}}(q)=\sum_{k=1}^{j} p_{0}^{k q} S_{k-j}^{K_{2}}(q)
$$

Analogously,

$$
S_{j}^{K_{1}^{\prime}}(q)=\sum_{k=1}^{j} p_{m}^{k q} S_{k-j}^{K_{2}^{\prime}}(q)
$$

Noting that $p_{m}^{k q} \leq p_{0}^{k q}, K_{2} \cup K_{2}^{\prime}=K_{3}$ and $S_{j}^{K}(q)$ is increasing in $K$, we obtain from (26) that

$$
\sum_{k=0}^{j} p_{0}^{k q} S_{j-k}^{K_{4}}(q) \leq \bar{S}_{j}(q) \leq 2 \sum_{k=0}^{j} p_{0}^{k q} S_{j-k}^{K_{3}}(q) .
$$

Fix now $\varepsilon>0$. Recall from the previous step that the $L^{q}$-spectrum of $\left.\mu\right|_{K_{i}}(i=3,4)$ exists and it is given by $\hat{\tau}$; moreover, in the course of the proof we showed that

$$
\hat{\tau}(q)=\lim _{k \rightarrow \infty} \delta k^{-1} \log S_{k}^{K_{i}}(q) .
$$

Recalling that $\delta=-1 / \log d$ we obtain, after taking exponentials, that

$$
d^{-\hat{\tau}(q)}=\exp (-\hat{\tau}(q) \log d)=\lim _{k \rightarrow \infty} \exp \left(\frac{\log S_{k}^{K_{i}}(q)}{k}\right)=\lim _{k \rightarrow \infty}\left(S_{k}^{K_{i}}(q)\right)^{1 / k} .
$$

Therefore we see that there is a constant $C=C(\varepsilon)>0$ such that

$$
C^{-1} d^{-k(\hat{\tau}(q)+\varepsilon)} \leq S_{k}^{K_{4}}(q) \leq S_{k}^{K_{3}}(q) \leq C d^{-k(\hat{\tau}(q)-\varepsilon)},
$$


for all $k \geq 0$ (the second inequality follows from the fact that $K_{4} \subset K_{3}$ ). Plugging this into (27) and adding we obtain

$$
C^{-1} \frac{p_{0}^{q(j+1)}-d^{-(j+1)(\hat{\tau}(q)+\varepsilon)}}{p_{0}^{q}-d^{-(\hat{\tau}(q)+\varepsilon)}} \leq \bar{S}_{j}(q) \leq 2 C \frac{p_{0}^{q(j+1)}-d^{-(j+1)(\hat{\tau}(q)-\varepsilon)}}{p_{0}^{q}-d^{-(\hat{\tau}(q)-\varepsilon)}} .
$$

Taking logarithms we get

$$
C^{\prime}\left(p_{0}^{q(j+1)}-d^{-(j+1)(\hat{\tau}(q)+\varepsilon)}\right) \leq \bar{S}_{j}(q) \leq C^{\prime \prime}\left(p_{0}^{q(j+1)}-d^{-(j+1)(\hat{\tau}(q)-\varepsilon)}\right)
$$

for some positive $C^{\prime}, C^{\prime \prime}$ independent of $j$.

Assume first than $p_{0}^{q}<d^{\hat{\tau}(q)}$. If $\varepsilon$ is so small that $p_{0}^{q}<d^{-(\hat{\tau}(q)+\varepsilon)}$ then, by taking logarithms and then the limit as $j \rightarrow \infty$ (while keeping $\varepsilon$ fixed) we deduce

$$
\hat{\tau}(q)-\varepsilon \leq \liminf _{j \rightarrow \infty} \frac{\log \bar{S}_{j}(q)}{-j \log d} \leq \limsup _{j \rightarrow \infty} \frac{\log \bar{S}_{j}(q)}{-j \log d} \leq \hat{\tau}(q)+\varepsilon .
$$

Recalling Proposition 1.2 and letting $\varepsilon \rightarrow 0$ we obtain $\hat{\tau}(q)=\tau(q)$ in this case.

Analogously, if $p_{0}^{q}<d^{\hat{\tau}(q)}$ then, recalling that $\bar{\alpha}=\log \left(p_{0}\right) /(-\log d)$ or $p_{0}=d^{-\bar{\alpha}}$, we get

$$
\bar{\alpha} q=\lim _{j \rightarrow \infty} \delta j^{-1} \log \bar{S}_{j}(q),
$$

or, in other words, $\tau(q)=\bar{\alpha} q$. In short, we have

$$
\tau(q)=\left\{\begin{array}{lll}
\bar{\alpha} q & \text { if } \quad \bar{\alpha} q>\hat{\tau}(q) \\
\hat{\tau}(q) & \text { if } \quad \bar{\alpha} q \leq \hat{\tau}(q)
\end{array} .\right.
$$

(The continuity of $\hat{\tau}$ guarantees that the formula above is also valid when $\hat{\tau}(q)=$ $\bar{\alpha} q)$. Assume first that $\alpha^{*}<\bar{\alpha}$. Since, by Theorem 1.1, $\log \eta(b, \sigma)<k \alpha^{*}$ if $\sigma \in \Xi_{k-1}$, and the number of terms in the sum $\hat{S}_{k}(q)$ is bounded by $C d^{k}$, we obtain that

$$
\hat{S}_{k}(q) \leq C d^{k} \exp \left(q k \alpha^{*}\right) \quad(q<0),
$$

and from here it follows that $\hat{\tau}(q) \geq q \alpha^{*}-1$ for negative $q$. Since $\hat{\tau}(0)=-1<0$, the concave curve $\hat{\tau}(q)$ meets the line $\bar{\alpha} q$ at a single negative point $q_{0}$, so (8) is verified.

It remains to handle the case $\alpha^{*}=\bar{\alpha}$. We have shown in the proof of Theorem 1.4 that

$$
\alpha^{*}=\delta \inf \left\{k^{-1} \log \eta(b, \sigma): \sigma \in \Xi_{k-1}\right\},
$$

whence $\hat{\tau}(q) \geq q \alpha^{*}=q \bar{\alpha}$ for all $q$. Recalling (28) we see that in this case $\tau(q)=\hat{\tau}(q)$ for all $q \in \mathbb{R}$. The proof is now complete.

Proof of Theorem 1.6. We continue using the notation of the proof of Theorem 1.4. From the multifractal theory for self similar sets under strong separation (see [Fal97], Theorem 11.5), it follows that the $L^{q}$-spectrum of $\mu^{k}$ is given by $T_{k}(q)=\hat{\tau}_{k}\left(\beta_{k} q\right)$, and the multifractal spectrum equals the Legendre transform of $T_{k}(q)$. Thus

$$
f_{H}(\alpha) \geq \operatorname{dim}_{H}\left\{x: \operatorname{dim} \mu^{k}(x)=\beta_{k} \alpha\right\}=T_{k}^{*}\left(\beta_{k} \alpha\right)=\hat{\tau}_{k}^{*}(\alpha) .
$$


The last equality follows from the definition of Legendre transform:

$$
T_{k}^{*}\left(\beta_{k} \alpha\right)=\inf _{q \in \mathbb{R}} q \beta_{k} \alpha-T_{k}(q)=\inf _{q^{\prime} \in \mathbb{R}} q^{\prime} \alpha-\hat{\tau}_{k}\left(q^{\prime}\right)=\hat{\tau}_{k}^{*}(\alpha) .
$$

Recall from Theorem 1.5 that $\hat{\tau}=\inf _{k} \hat{\tau}_{k}$. Let $F=\sup \hat{\tau}_{k}^{*}$. The subadditivity used in the first step of the proof of Theorem 1.4 shows that $F$ can be obtained as a monotone supremum of concave functions, and thus it is concave. Hence

$$
\begin{aligned}
\hat{\tau}_{k}^{*} \leq F \text { for all } k & \Rightarrow \hat{\tau}_{k} \geq F^{*} \text { for all } k \\
& \Rightarrow \hat{\tau} \geq F^{*} \Rightarrow \hat{\tau}^{*} \leq F
\end{aligned}
$$

where we used that the Legendre transform is involutive (i.e. $\left.\left(g^{*}\right)^{*}=g\right)$ ) and orderreversing on concave functions. We conclude that $f_{H}(\alpha) \geq F(\alpha) \geq \hat{\tau}^{*}(\alpha)$.

Let $I=d^{-1}(b, b+\xi)$, and denote by $\hat{f}_{H}$ the multifractal spectrum of $\left.\mu\right|_{I}$. We claim that $\hat{f}_{H} \geq f_{H}$ (the opposite inequality is obvious, but it is not what we need). To this end, observe that if $\omega \notin\{(0,0, \ldots),(m, m, \ldots)\}$ then $\operatorname{dim} \mu(\pi(b, \omega))=\operatorname{dim} \mu(\pi(\omega))$ (we have previously used both inequalities separately; they follow from Propositions 2.3 and 2.4). Thus $g:(0, \xi) \rightarrow I$ defined by $g(x)=d^{-1}(x+b)$ is a bi-Lipschitz map such that $\operatorname{dim} \mu(x)=\operatorname{dim} \mu(g(x))$. The claim now follows from the invariance of Hausdorff dimension under bi-Lipschitz maps.

We know from Theorem 1.5 that $\hat{\tau}$ is the $L^{q}$-spectrum of $\left.\mu\right|_{\bar{I}}$. We recall the result of Lau and Ngai ([LN99], Theorem 4.1), that the Legendre transform of the $L^{q}$-spectrum is always an upper bound for the multifractal spectrum; we remark that they do not assume self-similarity. Hence we are able to conclude that $f_{H}(\alpha) \leq \hat{\tau}^{*}(\alpha)$, and this completes the proof.

Proof of Proposition 1.7. Let $g_{k}: \mathcal{A}^{\mathbb{N}} \rightarrow \mathbb{R}$ be given by

$$
g_{k}(\omega)=-\log \eta(\omega \mid k)
$$

Observe that

$$
\int_{\mathcal{A}^{N}} g_{k}(\omega) d \nu=\sum_{\sigma \in \mathcal{A}^{k}}-\mathbf{p}(\sigma) \log \eta(\sigma)=\sum_{\sigma \in \Xi_{k}}-\eta(\sigma) \log \eta(\sigma) \geq 0 .
$$

Note that Lemma 2.1 can be restated as $g_{n+k}(\omega) \leq g_{n}(\omega)+g_{k}\left(T^{n}(\omega)\right)$ (recall that $T$ is the shift operator). Hence the fact that the local dimension exists and is almost everywhere constant, as well as the first equality in the proposition, follow from Kingman's subadditive ergodic theorem [Kin68] applied to the system $\left(\mathcal{A}^{\mathbb{N}}, \nu_{\mathbf{p}}, T,\left\{g_{k}\right\}_{k}\right)$.

The second equality follows analogously, by considering the functions $h_{k}: \mathcal{A}^{\mathbb{N}} \rightarrow \mathbb{R}$ given by

$$
h_{k}(\omega)=-\log \|M(\omega \mid k)\|,
$$

for some fixed consistent norm $\|\cdot\|$, and applying the subadditive ergodic theorem to the family $\left\{-h_{k}\right\}$.

4. Examples and applications. A consequence of Theorems 1.5 and 1.6 is that, in the case $m<2 d-2$, the multifractal formalism holds for $\mu$ if and only if $\alpha^{*}=\bar{\alpha}$. Therefore it is of interest to find explicit necessary and sufficient conditions for the equality of $\alpha^{*}$ and $\bar{\alpha}$; the next proposition does precisely this. 
Proposition 4.1. $\alpha^{*}=\bar{\alpha}$ if and only if $m \leq 2 d-2$ and $p_{0}=p_{i}$ for some $i \in\{m-d+1, \ldots, d-1\}$.

Proof. Assume first that $p_{0}=p_{i}$ for some $i \in\{m-d+1, \ldots, d-1\}$ (whence, in particular, $m \leq 2 d-2)$. In this case $a=1$ and an inspection of the matrix $M_{i}$ shows that $\rho\left(M_{i}\right)=p_{0}$ (the eigenvalues are $p_{i}$ and $p_{0}$ or $p_{m}$ or both; but we are assuming that these numbers are equal). Hence

$$
\alpha^{*} \leq \operatorname{dim} \mu(\pi(i, i, \ldots))=\delta \log \rho\left(M_{i}\right)=\bar{\alpha}
$$

and $\alpha^{*}=\bar{\alpha}$ in this case.

Assume now that $p_{0}<p_{i}$ for $i=m-d+1, \ldots, d-1$ (if there is any such $i$ ). Without loss of generality we assume $m>d$. A simple modification of Proposition 3.4 of [HL01] shows the following: for every $s \in(0, \xi)$ there is $\omega \in \mathcal{A}^{\mathbb{N}}$ such that $\pi(\omega)=s$ and the digits 0 and $m$ appear in $\omega$ only finitely many times. Since shifting such $\omega$ does not change upper and lower local dimensions, we obtain that

$$
\alpha^{*} \leq \sup \left\{\overline{\operatorname{dim}} \mu(\pi(\omega)): \omega \in\{1, \ldots, m-1\}^{\mathbb{N}}\right\} .
$$

We will show that $\eta(\sigma)>p_{0}^{2}$ for every $\sigma \in\{1, \ldots, m-1\}^{2}$. Indeed, if $\sigma_{2} \notin\{m-d+$ $1, \ldots, d-1\}$ let

$$
\sigma^{*}=\left\{\begin{array}{lll}
\left(\sigma_{1}+1, \sigma_{2}-d\right) & \text { if } & \sigma_{2} \geq d \\
\left(\sigma_{1}-1, \sigma_{2}+d\right) & \text { if } & \sigma_{2} \leq m-d
\end{array} .\right.
$$

Note that $\pi(\sigma)=\pi\left(\sigma^{*}\right)$, whence

$$
\eta(\sigma) \geq \mathbf{p}(\sigma)+\mathbf{p}\left(\sigma^{*}\right) \geq 2 p_{0}^{2}
$$

If, on the other hand, $\sigma_{2} \in\{m-d+1, \ldots, d-1\}$, then

$$
\eta(\sigma) \geq \mathbf{p}(\sigma) \geq p_{0} \min \left\{p_{m-d+1}, \ldots, p_{d-1}\right\}
$$

Thus in any case $\eta(\sigma) \geq \tilde{p} p_{0}$, where

$$
\tilde{p}=\min \left\{p_{m-d+1}, \ldots, p_{d-1}, 2 p_{0}\right\}>p_{0} .
$$

Splitting $\omega \mid 2 k$ in $k$ chunks of length 2 and using supermultiplicativity we get $\eta(\omega \mid 2 k) \geq$ $\left(\tilde{p} p_{0}\right)^{k}$, whence

$$
\overline{\operatorname{dim}} \mu(\pi(\sigma)) \leq \delta \frac{\log \tilde{p}+\log p_{0}}{2}<\delta \log p_{0}=\bar{\alpha}
$$

Thus $\alpha^{*}<\bar{\alpha}$ in this case, completing the proof. $\square$

The case in which all the weights are equal is interesting for several reasons. On one hand, it is the most "purely combinatoric" case; on the other, the vector $\mathbf{p}$ is extremal among all allowed weights of the same length. We investigate this case more closely. We begin by showing that if $m \equiv-1 \bmod d$, then $\mu$ is absolutely continuous.

Proposition 4.2. Let $m=n k-1$ for some $n \geq 2$, and take $p_{i}=1 /(m+1)$ for all $i$. The measure $\mu$ thus obtained is absolutely continuous with a bounded density. 
Proof. From the proof of Proposition 2.4 it follows that $a$ can be replaced by any integer greater or equal than $\lfloor(m-d) /(d-1)\rfloor$. For the purposes of this proof we will take $a=\lfloor 1+\xi\rfloor$. Recall that

$$
\mu\left(B\left(\pi(\omega), d^{-k}\right)\right) \leq \mu_{k}\left(B\left(\pi(\omega \mid k),(1+\xi) d^{-k}\right)\right) .
$$

By the choice of $a$, the right hand side is equal to the central column of $M(\sigma \mid k)$ (this also follows from the proof of Proposition 2.4). Hence

$$
\mu\left(B\left(\pi(\omega), d^{-k}\right)\right) \leq\|M(\omega \mid k)\|_{1} \leq C_{1}\|M(\omega \mid k)\|_{\infty} \leq C_{1}\left(\max _{0 \leq i \leq m}\left\|M_{i}\right\|_{\infty}\right)^{k} .
$$

for some constant $C_{1}$. But the $\infty$-operator norm is equal to the maximum of the 1-norms of the rows. Observe that any given row of $M_{i}$ is of the form $\left(p_{j-a d}, \ldots, p_{j}, \ldots, p_{j+a d}\right)$, whence at most $n$ of the coordinates are nonzero. Since $p_{j}=(d n)^{-1}$ for every $j=0, \ldots, m$, it follows that $\left\|M_{i}\right\|_{\infty} \leq d^{-1}$ for all $i=0, \ldots, m$. Thus we obtain that

$$
\mu(B(\pi(\omega), r)) \leq C_{2} \lambda(B(\pi(\omega), r)) \quad \text { for every } \omega \in \mathcal{A}^{\mathbb{N}}, r>0,
$$

for some constant $C_{2}$, where $\lambda$ denotes Lebesgue measure on the line. We conclude that $\mu$ is absolutely continuous and, moreover, $d \mu / d \lambda$ is bounded. $\square$

The next proposition deals with the case $m=d$.

Proposition 4.3. Let $m=d$ and $p_{i}=1 /(m+1)$ for all $i$. Then

$$
\underline{\alpha}=\frac{\log (d+1)-\log \zeta}{\log d},
$$

where $\zeta=(1+\sqrt{5}) / 2$ is the golden number. Also $\alpha^{*}=\bar{\alpha}=\log (d+1) / \log d$.

Proof. A calculation shows that

$$
M_{0} M_{1}=\frac{1}{(d+1)^{2}}\left(\begin{array}{lll}
1 & 1 & 0 \\
1 & 2 & 0 \\
0 & 1 & 0
\end{array}\right) \Longrightarrow \rho\left(M_{0} M_{1}\right)=\left(\frac{\zeta}{d+1}\right)^{2} .
$$

whence

$$
\underline{\alpha} \leq \frac{\delta \log \left(\rho\left(M_{0} M_{1}\right)\right)}{2}=\frac{\log (d+1)-\log \zeta}{\log d} .
$$

We will inductively show that for all $\sigma \in \mathcal{A}^{j}$,

$$
\begin{array}{ll}
\sigma_{j} \in\{0, d\} & \Rightarrow \eta(\sigma) \leq(d+1)^{-j} F_{j} \\
\sigma_{j} \in\{1, \ldots, d-1\} & \Rightarrow \eta(\sigma) \leq(d+1)^{-j} F_{j-1},
\end{array}
$$

where $F_{j}$ denotes the $j$-th Fibonacci number. Indeed, this is clear for $j=1,2$. Assume it is valid for $j=1, \ldots, n$, and let $\sigma \in \mathcal{A}^{n+1}$. Observe that if $\sigma^{\prime} \in[\pi(\sigma)]_{n+1}$ then $\sigma_{n+1}^{\prime} \equiv \sigma_{n+1} \bmod d$ (just multiply $\pi(\sigma)=\pi\left(\sigma^{\prime}\right)$ by $\left.d^{n+1}\right)$. Hence two cases arise. If $0<\sigma_{n+1}<d$ we obtain

$$
\eta(\sigma)=\eta(\sigma \mid n) p_{\sigma(n+1)} \leq(d+1)^{-n} F_{n},
$$


by the inductive hypothesis. If $\sigma_{n+1}=0$ and $\sigma^{\prime} \in[\pi(\sigma)]_{n+1}$, it must be $\sigma_{n+1}^{\prime}=0$ or $d$. In the second case $\pi(\sigma \mid n)=\pi\left(\sigma^{\prime} \mid n\right)+d^{-n}$ whence, multiplying by $d^{n}, \sigma_{n} \equiv \sigma_{n}^{\prime}+1$ $\bmod d$. It follows that $\sigma_{n}$ and $\sigma_{n}^{\prime}$ cannot be simultaneously in $\{0, d\}$, and therefore, using the inductive hypothesis,

$$
\eta(\sigma)=p_{0} \eta(\sigma \mid n)+p_{m} \eta\left(\sigma^{\prime} \mid n\right) \leq(d+1)^{-n}\left(F_{n-1}+F_{n-2}\right)=(d+1)^{-n} F_{n} .
$$

(if no such $\sigma^{\prime}$ exists it is even simpler to obtain the needed estimate). The case $\sigma_{n+1}=d$ is handled in the same way. that

Recall that $F_{j}=\zeta^{j}-\zeta^{\prime j}$ for some $0<\zeta^{\prime}<1$. We conclude from Proposition 2.3

$$
\underline{\operatorname{dim}} \mu(\pi(\omega)) \geq \liminf _{j \rightarrow \infty} \frac{\log \left((d+1)^{-j} F_{j}\right)}{j \log d}=\frac{\log (d+1)-\log \zeta}{\log d} .
$$

This yields the other inequality for $\underline{\alpha}$. The rest of the proposition follows immediately from Proposition 4.1. $\mathrm{c}$

Another interesting class of examples are the convolutions of certain Cantor measures. The next lemma is standard but we include the computation for completeness.

Lemma 4.4. Let $\left(p_{0}, \ldots, p_{m}\right)$ and $\left(p_{0}^{\prime}, \ldots, p_{m^{\prime}}^{\prime}\right)$ be two probability vectors. Denote by $\mu$ and $\mu^{\prime}$ the attractors of the IFS

$$
\left\{\left(\frac{x}{d}+\frac{i}{d}, p_{i}\right): 0 \leq i \leq m\right\}, \quad\left\{\left(\frac{x}{d}+\frac{i}{d}, p_{i}^{\prime}\right): 0 \leq i \leq m^{\prime}\right\}
$$

(we are not assuming any condition on the weights, $p, m$ or $m^{\prime}$ ). Then $\mu * \mu^{\prime}$ is the attractor of the IFS

$$
\left\{\left(\frac{x}{d}+\frac{i}{d}, v_{i}\right): 0 \leq i \leq m+m^{\prime},\right\}
$$

where

$$
v_{i}=\sum\left\{p_{j} p_{j^{\prime}}^{\prime}: 0 \leq j \leq m, 0 \leq j^{\prime} \leq m^{\prime} \text { and } j+j^{\prime}=i\right\}
$$

Proof. Write $\phi_{i}(x)=x / d+i / d, S(x, y)=x+y$, and compute

$$
\begin{aligned}
\mu * \mu^{\prime}(A) & =\mu \times \mu^{\prime}\left(S^{-1}(A)\right) \\
& =\sum_{i, j} p_{i} p_{j}^{\prime} \mu \times \mu^{\prime}\left(\left(S \circ \phi_{i} \times \phi_{j}\right)^{-1}(A)\right) \\
& =\sum_{i, j} p_{i} p_{j}^{\prime} \mu \times \mu^{\prime}\left(\left(\phi_{i+j} \circ S\right)^{-1}(A)\right) \\
& =\sum_{i, j} p_{i} p_{j}^{\prime}\left(\mu * \mu^{\prime}\right) \phi_{i+j}^{-1}(A) .
\end{aligned}
$$

The lemma follows.

Note that the above lemma also shows that the class of measures studied here is closed under convolution. We will now briefly consider the convolutions of biased (middle-third) Cantor measures. Fix $0<p \leq 1 / 2$, and let $\mu_{p}^{0}$ be the attractor of the IFS $\{(x / 3, p),((x+1) / 3,1-p)\}$. The lemma shows that if we let $p_{i}=\left(\begin{array}{c}m \\ i\end{array}\right)(1-a)^{i} a^{m-i}$ and $d=3$, then $\mu$ is the $m$-fold convolution of $\mu_{p}^{0}$; note, however, that the resulting 
weight vector $\mathbf{p}$ is not always regular. Since we are assuming $p \leq 1 / 2$, the minimum non-extreme weight is $m p^{m-1}(1-p)$; it follows that $\mathbf{p}$ is regular if and only if

$$
(1-p)^{m} \leq m p^{m-1}(1-p) \quad \Longleftrightarrow \quad p \geq \frac{1}{1+m^{\frac{1}{m-1}}} .
$$

The numerical value of the minimal $p$ that makes $\mathbf{p}$ regular for $m=3$ is 0.366025 .

Let $\mu_{k}$ be the $k$-fold convolution of the standard Cantor measure. We know from Theorem 1.1 that $\max \Delta \mu_{k}=k \log 2 / \log 3$; because of Proposition 4.1, this maximum local dimension is isolated. Let

$$
\begin{aligned}
\underline{\alpha}_{k} & =\inf \Delta \mu_{k} ; \\
\alpha_{k}^{*} & =\sup \Delta \mu_{k} \backslash\{k \log 2 / \log 3\} ; \\
\gamma_{k} & =\operatorname{dim}_{H}\left(\mu_{k}\right) .
\end{aligned}
$$

In [HL01] the authors computed the exact values of $\alpha_{3}^{*}, \underline{\alpha}_{3}$ and $\underline{\alpha}_{4}$. Here we compute the value of $\alpha_{4}^{*}$ :

LEMMA 4.5.

$$
\alpha_{4}^{*}=\frac{\log (16 / 5)}{\log 3}=1.05875 \ldots
$$

and this dimension is attained at $x=1 / 2=\pi(1,1, \ldots)$

Proof. A calculation shows that $\rho(M(1))=\log (16 / 5) / \log 3$ and therefore, by Lemma 2.6, $\operatorname{dim} \mu_{4}(1 / 2)=\log (16 / 5) / \log 3$.

We claim that

$$
\sigma_{1} \notin\{0,4\} \Longrightarrow \eta(\sigma) \geq \frac{1}{5}\left(\frac{5}{16}\right)^{|\sigma|}
$$

Assuming the claim, the lemma follows at once from the previous calculation and Corollary 3.1.

The claim will be proved by induction in $|\sigma|$. It is clear for $|\sigma|=1$. Now let $|\sigma|=j+1>1$ and assume the case $|\sigma|=j$ has been verified. If $\sigma_{j+1}=0$ then $\sigma$ can also be represented by a sequence ending in 3: since $\sigma_{j+1}=0$ and $\sigma_{1}>0, \pi(\sigma)=i 3^{-j}$ for some positive integer $i$. Then if $\sigma^{\prime} \in \mathcal{A}^{j}$ is such that $\pi\left(\sigma^{\prime}\right)=(i-1) 3^{-j}$, we get $\pi(\sigma)=\pi\left(\sigma^{\prime}, 3\right)$. Therefore

$$
\eta(\sigma) \geq\left(p_{0}+p_{3}\right) \min \left\{\eta\left(\sigma^{\prime}\right): \sigma^{\prime} \in \mathcal{A}^{j}, \sigma_{1} \notin\{0,4\}\right\} \geq \frac{5}{16} \frac{1}{5}\left(\frac{5}{16}\right)^{j},
$$

using the inductive hypothesis and the values $p_{0}=1 / 16 ; p_{3}=4 / 16$. A similar argument holds if $\sigma_{j+1}=1,3$ or 4 . If $\sigma_{j+1}=2$ then the same conclusion is still true since $p_{2}=6 / 16>5 / 16$. In any case, the next step is verified and the lemma follows.

We remark that although there is no barrier digit for the 4 -fold convolution of the Cantor measure we can still prove that

$$
\Delta \mu_{4}=\left[\underline{\alpha}_{4}, \alpha_{4}^{*}\right] \cup\left\{\bar{\alpha}_{4}\right\} .
$$

The idea is as follows: let $\sigma^{k, l}$ be the sequence consisting of $k$ twos followed by $l$ ones. By computing the matrices $M\left(\sigma^{k, l}\right)$ explicitly one can show that the local dimensions 
arising from such sequences are dense in $\left[\underline{\alpha}_{4}, \alpha_{4}^{*}\right]$; by an approximation argument one can show that actually all intermediate dimensions occur.

With the help of Theorem 1.1 (or more precisely Corollary 3.1 ) it is possible to estimate the values of $\underline{\alpha}_{k}$ and $\alpha_{k}^{*}$ for other values of $k$, as well as the corresponding parameters in the biased case. It turns out that it is easier to obtain good estimates for $\underline{\alpha}_{k}$; The following table summarizes these estimates for $5 \leq k \leq 10$. As is to be expected, the smallest local dimensions approach 1 as $k$ increases, reflecting the progressive smoothing produced by the successive convolutions.

\begin{tabular}{|c|c|c|}
\hline$k$ & $\underline{\alpha}_{k}$ (l.b.) & $\underline{\alpha}_{k}$ (u.b.) \\
\hline 5 & 0.972510 & 0.972638 \\
\hline 6 & 0.976057 & 0.976628 \\
\hline 7 & 0.993697 & 0.993848 \\
\hline 8 & 0.994940 & 0.995246 \\
\hline 9 & 0.998585 & 0.998657 \\
\hline 10 & 0.998908 & 0.999022 \\
\hline
\end{tabular}

We remark that in [LN00] the value of $\gamma_{3}$ was computed with 4 decimal digits of accuracy (In fact, what they computed is the value of $\tau^{\prime}(1)$ where $\tau$ is the $L^{q}$-spectrum of the 3 -fold convolution of the Cantor measure; but it is known that this is equal to the Hausdorff dimension when the measure has an almost sure local dimension, which is the case by Proposition 1.7). Theoretically, it is possible to use Proposition 1.7 to estimate the value of $\gamma_{k}$, but unfortunately that seems to require extreme computing power (it is not hard to obtain an accuracy of two decimal digits, but since for $k \geq 4$ the values appear to be between 0.99 and 1 , this is rather meaningless).

5. Remarks and open questions. We finish the paper with some remarks on the relationship between our results and other recent research in the multifractal theory of self-similar measures.

1. The measures studied here verify the "weak separation condition" (w.s.c.) introduced in [LN99]. The main result of that paper is that, under the w.s.c., $f(\alpha)=\tau^{*}(\alpha)$ for any $\alpha=\tau^{\prime}(q), q \geq 0$. The authors were able to check the differentiability of $\tau(q)$ in the range $q \geq 0$ for some concrete classes of measures, including a small subset of the measures analyzed in this paper [LN00]. Our examples show that $\tau(q)$ may not be differentiable for $q<0$, even when $\mu$ is singular. Moreover, this leads to the failure of the multifractal formalism, as $\tau(q)$ does not "see" an interval of local dimensions. However, it remains a challenging open question whether $\tau(q)$ is differentiable in $[0, \infty)$ for every weakly-separated self-similar measure.

2. Olsen [Ols95] introduced a more general multifractal framework. He considers several "coarse" and "fine" versions of both the multifractal and $L^{q}$-spectra; the coarse versions are defined in terms of coverings or packings by balls of the same radius, while in the fine spectra variable radii are allowed. Could it be that the failure of the multifractal formalism in our setting is due to our consideration of inappropriate (non-matching) versions of the relevant spectra, rather than to an intrinsic characteristic of the measures? Even if this were the case, it would be in sharp contrast with the non-overlapping situation, in which all of the spectra coincide. We believe, however, that the breakdown 
of the multifractal formalism is essential, and should be corroborated by any reasonably-defined version of the spectra.

3. When a barrier digit $b$ is present, the self-similar measure $\mu$ can also be obtained as the attractor of an infinite iterated function system without overlaps. Indeed, for $\sigma \in \mathcal{A}^{k}$ write

$$
\bar{\sigma}=[\pi(\sigma)]_{k} ; \quad \Omega=\left\{\overline{(\sigma, b)}: \sigma \in(\mathcal{A} \backslash\{b\})^{*}\right\} .
$$

Let

$$
\phi(\bar{\sigma})(x)=\frac{x}{d^{|\sigma|}}+\pi(\sigma)
$$

it is clear that the definition is independent of the representative chosen. Define also

$$
\mathcal{I}=\{(\phi(\bar{\sigma}), \eta(\sigma)): \bar{\sigma} \in \Omega\} .
$$

It is easy to see that $\mu$ is the attractor of the infinite IFS $\mathcal{I}$ and, since $b$ is a barrier, the open set condition is verified (take $(0, \xi)$ as the open set). The multifractal theory for such (and far more general) infinite IFS was developed in [HMU02], where the multifractal formalism was shown to hold in certain region, depending on some conditions. Since we are mainly concerned with the region where the multifractal formalism fails, we have not attempted to use the results of [HMU02].

4. The results of this paper give no information about whether the extreme local dimensions $\underline{\alpha}$ and $\alpha^{*}$ are attained. We do not know of any example where either of them is not attained, and we conjecture that this cannot happen.

5. Although our methods do not seem to generalize to other self-similar measures, our results may still hold in greater generality. In particular, the following question arises naturally: let $\mu$ be a self-similar measure on $\mathbb{R}^{n}$ whose attractor has nonempty interior. Let $K$ be a compact subset of the interior of $\operatorname{supp} \mu$. Is it always true that $\left.\mu\right|_{K}$ verifies the multifractal formalism?

We conjecture that the answer is affirmative, at least in the case where $\operatorname{supp} \mu$ is a linear interval.

Acknowledgments. The author gratefully thanks Doctor Ursula Molter for many valuable discussions, suggestions and comments on early version of the paper. He also would like to thank Professor Boris Solomyak for some helpful conversations and for pointing to some relevant literature.

\section{REFERENCES}

[AP96] Matthias Arbeiter And Norbert Patzschke, Random self-similar multifractals, Math. Nachr., 181 (1996), pp. 5-42.

[BW92] Marc A. Berger ANd YAng WAng, Bounded semigroups of matrices, Linear Algebra Appl., 166 (1992), pp. 21-27.

[CM92] R. CAWLEy AND D. MAULDIn, Multifractal decompositions of moran fractals, Advances in Mathematics, 92 (1992), pp. 192-236.

[Fal97] K.J. FALCONER, Techniques in Fractal Geometry, John Wiley \& Sons, 1997.

[HL01] Tian-You Hu and Ka-Sing Lau, Multifractal structure of convolution of the Cantor measure, Adv. in Appl. Math., 27:1 (2001), pp. 1-16.

[HMU02] P. Hanus, R. D. Mauldin, And M. Urbański, Thermodynamic formalism and multifractal analysis of conformal infinite iterated function systems, Acta Math. Hungar., 96(1-2) (2002), pp. 27-98. 
[Kin68] J. F. C. Kingman, The ergodic theory of subadditive stochastic processes, J. Roy. Statist. Soc. Ser. B, 30 (1968), pp. 499-510.

[Lau99] KA-SING LAU, Iterated function systems with overlaps and multifractal structure, In Trends in probability and related analysis (Taipei, 1998), pages 35-76. World Sci. Publishing, River Edge, NJ, 1999.

[LN99] K.S. LAU AND S.M. NGaI, Multifractal measures and a weak separation condition, Advances in Mathematics, 141 (1999), pp. 45-06.

[LN00] K.S. LAU AND S.M. NGaI, Second-order self-similar identities and multifractal descompositions, Indiana Univ. Math. J., 49 (2000), pp. 925-972.

[LW95] JefFREY C. LAGARIAS AND YANG WANG, The finiteness conjecture for the generalized spectral radius of a set of matrices, Linear Algebra Appl., 214 (1995), pp. 17-42.

[Ols95] L. Olsen, A multifractal formalism, Advances in Mathematics, 116 (1995), pp. 82-196.

[Pes97] Y. Pesin, Dimension Theory in Dynamical Systems, Chicago Lectures in Tathematics, The University of Chicago Press, 1997.

[PS00] Yuval Peres and Boris Solomyak, Existence of $L^{q}$ dimensions and entropy dimension for self-conformal measures, Indiana Univ. Math. J., 49:4 (2000), pp. 1603-1621. 